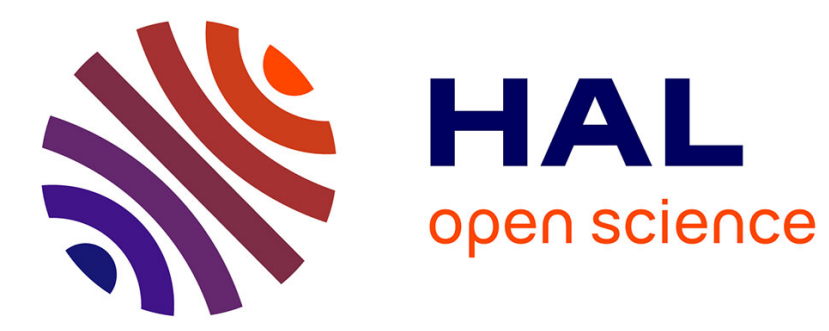

\title{
Sur les propriétés des gaz très purs au voisinage du point critique
}

P. Villard

\section{To cite this version:}

P. Villard. Sur les propriétés des gaz très purs au voisinage du point critique. J. Phys. Theor. Appl., 1894, 3 (1), pp.441-451. 10.1051/jphystap:018940030044100 . jpa-00239825

\section{HAL Id: jpa-00239825 https://hal.science/jpa-00239825}

Submitted on 1 Jan 1894

HAL is a multi-disciplinary open access archive for the deposit and dissemination of scientific research documents, whether they are published or not. The documents may come from teaching and research institutions in France or abroad, or from public or private research centers.
L'archive ouverte pluridisciplinaire HAL, est destinée au dépôt et à la diffusion de documents scientifiques de niveau recherche, publiés ou non, émanant des établissements d'enseignement et de recherche français ou étrangers, des laboratoires publics ou privés. 


\section{SUR LES PROPRIÉTÉS DES GAZ TRÈS PURS AO VOISINAGE DU POINT GRITIQUE;}

Par M. P. VILlard.

Depuis les découvertes de Cagniard de Latour et d'Andrews, de nombreux travaux ont été effectués en vue de constater la généralité des phénomènes observés par ces deux physiciens, en vue surtout de vérifier les conséquences qui résultaient de leurs expériences.

Ainsi qu'on devait s'y attendre, et en raison des difficultés que comportent les recherches de ce genre, les résultats obtenus ont conduit les expérimentateurs à des conclusions souvent opposées, et de nature à compliquer une question qui se présentait tout d'abord avec un caractère de grande simplicité.

L'examen d'un réseau d'isothermes analogue à celui construit par Andrews pour l'acide carbonique montre immédiatement que la liquéfaction d'un gaz, représentée graphiquement par la partie rectiligne des isothermes, est un phénomène de moins en moins accusé au fur et à mesure que la température s'élève.

On arrive bientôt à une isotherme limite $\mathrm{T}_{c} \mathrm{~T}_{c}^{\prime}$ qui n'offre plus

Fig. I.

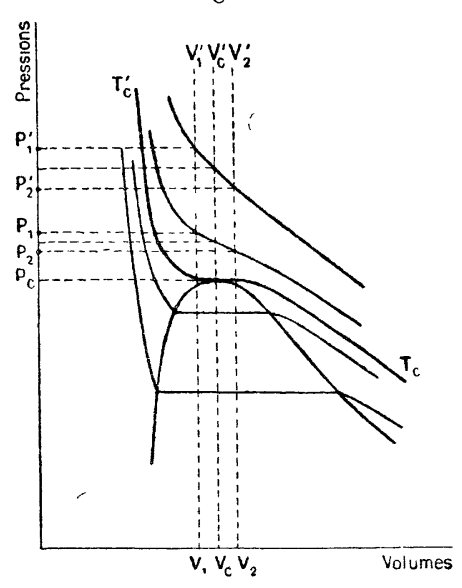

qu'un simple point d'inflexion à tangente horizontale. La température critique est atteinte, et à partir de ce moment les courbes J. de Phys., 3 série, t. III. (Octobre 1894 .) 
ne présentent plus ni discontinuité rectiligne, ni tangente parallèle à l'axe des volumes, mais seulement une inflexion qui va s'atténuant de plus en plus. Il semble donc qu'au delà de la température critique ainsi définie il n'y ait plus de liquéfaction possible; on doit alors se trouver toujours en présence d'un fluide homogène ne possédant qu'une série unique de propriétés, et en particulier qu'une seule densité.

Dans les expériences qui font l'objet de cet exposé, j'ai précisément cherché à mettre en évidence cette homogénéité mise en doute par divers physiciens.

Ramsay ( ${ }^{1}$ ) en Angleterre et Jamin ( ${ }^{2}$ ) en France ont émis l'idée qu'à la température critique la vaporisation n'était pas com plète, les densités du liquide et de sa vapeur étant néanmoins égales. Les conclusions de Jamin peuvent se résumer ainsi :

A partir d'une certaine température, la densité du liquide devient et reste égale à celle de sa vapeur : c'est le point critique. La chaleur latente n'existe plus, le liquide se mélange avec sa vapeur dont rien ne le différencie, et il cesse alors d'être visible; mais la loi générale de vaporisation n'est interrompue à aucun moment par un point critique quelconque (Comptes rendus des séances de l'Académie des Sciences, t. XCVI; p. I 448).

Cette hypothèse a été combattue en 889 par MM. Cailletet et Colardeau $\left({ }^{3}\right)$. Des expériences très ingénieuses les ont conduit à admettre qu'à la température de disparition du niveau et à plusieurs degrés au dessus il existait encore un liquide et une vapeur présentant des densités différentes, mais susceptibles de se dissoudre l'un dans l'autre en toutes proportions.

En 1892 et 1893 M. Zambiasi ( ${ }^{4}$ ) et M. Battelli ( ${ }^{5}$ ) sont arrivés au contraire à établir, en opérant avec l'éther, qu'au point critique il n'y avait qu'une seule densité, mais ils admettent que la vapo-

( $\left.{ }^{1}\right)$ Proceedings of the Roy. Soc., t. XXX, p. 323, et t. XXXI, p. 194; 1880.

( ${ }^{2}$ ) Comptes rendus des séances de l'Académie des Sciences, t. XGVI, p. 1448 ; I 883 et t. XCVII, p. 10. Journal de Physique, $2^{\circ}$ série, t. II, p. $393 ; 1883$.

( $\left.{ }^{3}\right)$ Comptes rendus des séances de l'Académie des Sciences, t. CVIII; p. I28o. Journal de Physique, $2^{\text {e }}$ série, t. VIII, p. 389 ; 1889. Annales de Chimie et de Physique, 6e série, t. XVIII ; p. 269.

(*) Atti della Reale Accademia dei Lincei, t. I, p. 423; $89^{2}$. Journal de Physique, $3^{\mathrm{e}}$ série, t. II.

( ${ }^{5}$ ) Annales de Chimie et de Physique, 6e série, t. XXIX, p. 400; $189^{3}$. 
risation continue aux températures supérieures, conformément à l'hypothèse de Jamin.

La conclusion qui semble se dégager de ces divers travaux est qu'à la température critique et au delà il existe encore deux fluides distincts.

Sans vouloir faire une étude complète des phénomènes critiques, j'ai repris quelques-unes des expériences imaginées à ce sujet, mais en ayant soin d'employer des gaz aussi purs que possible; c'est là une condilion dont l'importance se comprend immédiatement si l'on réfléchit que de l'acide carbonique, par exemple, contenant seulement un millième d'air en volume, est comparable comme pureté, une fois liquéfié, à de l'eau saturée d'air sous une pression de 18 atmosphères.

J'ai choisi pour ces recherches le protoxyde d'azote et l'acide carbonique dont le point critique est mieux connu, surtout depuis les travaux de M. Amagat (') et de M. Chappuis ( $\left.{ }^{2}\right)$.

J'indiquerai d'abord la manière d'obtenir ces gaz à un état de pureté convenable :

Protoxyde d'azote. - Je me suis servi, en le purifiant, du gaz que l'on trouve dans le commerce, liquéfié dans des récipients en fer. Destiné à des usages médicaux, il ne contient comme impurelés que de l'azote provenant de la préparation, un peu d'air el peut-être d'acide carbonique.

Au sortir du récipient, et après avoir franchi un robinet d'arrêt, le protoxyde traverse des tubes en cristal épais, de $10^{\mathrm{mm}}$ à $12^{\mathrm{mm}}$ de diamètre intérieur, contenant de la potasse, de la ponce sulfurique, de la potasse fondue, puis il se liquéfie dans un tube refroidi. La pression à laquelle a lieu la condensation s'élève peu à peu, indiquant nettement que l'azote et l'air s'accumulent dans l'atmosphère surmontant le liquide; il convient de chasser de temps en temps ce mélange gazeux et même de faire bouillir le protoxyde liquéfié. Cette première distillation terminée, on élimine,

( $\left.{ }^{1}\right)$ Comptes rendus des séances dè l'Académie des Sciences, t. CXIV, p. rog33. Journal de Physique, $3^{\text {e }}$ série, t. I, p. $288 ; 189^{2}$.

( $\left.{ }^{2}\right)$ Comptes rendus des séances de l'Académie des Sciences, t. CXVIII, p. $976 ; 189$ 4. 
par une ébullition prolongée, la majeure partie des gaz dissous, puis le tube est retourné et le liquide bouillant est dirigé, par la manœuvre d'un robinet, dans le tube définitif qui servira aux expériences; les premières portions servent à laver les parois et sont rejetées, puis le remplissage a lieu, suivi d'une dernière ébullition assez longue, après quoi on procède à la fermeture.

Dans la plupart des cas, afin d'avoir des appareils indépendants et faciles à manier, j'ai scellé au chalumeau les tubes remplis de gaz liquéfié; pour cela, un fragment de cire de masse négligeable (une fraction de milligramme) est placé d'avance à l'entrée d'une partie très capillaire, puis fondu au moment voulu. Cette obturation provisoire permet d'effectuer ensuite sans peine le scellage définitif. Mais la cire n'est pas nécessaire : si l'étranglement capillaire est assez fin, on peut couper le tube un peu au-dessus, et, pendant que le gaz s'échappe lentement, le cristal est fondu puis serré dans une pince froide et enfin recuit. Aucune trace d'air ne peut évidemment pénétrer et l'introduction d'une matière étrangère est évitée.

Toute cette rectification s'effectue dans un appareil absolument hermétique, sans joints par serrage, sans cuirs gras, les robinets eux-mêmes étant entièrement métalliques.

En une seule opération, j’ai pu obtenir ainsi jusqu'à $20^{\mathrm{gr}}$ de protoxyde d'azote purifié.

Acide carbonique. - Le même procédé a été appliqué à l'acide carbonique livré par l'industrie. Il est seulement nécessaire de laisser sortir des récipients le gaz et non le liquide qui est souillé par diverses impuretés. L'acide en vapeur traverse d'abord un tube à acide chromique humide divisé par de la pierre ponce, et se liquéfie dans un grand tube refroidi contenant du bicarbonate de sodium; après y avoir subi une ébullition suffisante, le liquide obtenu est redistillé et se rend dans les appareils définitifs en passant sur de la ponce sulfurique. L'opération se termine comme pour le protoxyde d'azote.

La pureté des produits obtenus ainsi ne peut être que difficilement constatée par l'analyse, surtout en ce qui concerne le protoxyde d'azote. Pour l'acide carbonique, l'absorption par la potasse n'a laissé, sur $\mathrm{I} 40^{\mathrm{cc}}$ de gaz, qu'un résidu certainement inférieur 
$\mathrm{I}^{\mathrm{mmc}}$. Dans ces conditions, l'analyse n'a aucun sens précis, et elle n'a rien gagné à être répétée avec $600^{\text {cc }}$.

Les propriétés physiques seules peuvent offrir un critérium suffisant.

Avec le protoxyde d'azote que j'avais étudié en premier lieu, j'ai constaté en particulier que la liquéfaction sans résidu se produisait dès que la pression dépassait de quelques centimètres de mercure la tension maxima. Le phénomène n'est pas rapide, il est vrai, mais si, au moment où il ne reste plus qu'une bulle gazeuse très petite, on promène celle-ci dans la masse parfois assez grande du liquide, on constate que sa disparition n'est pas sensiblement accélérée, comme cela aurait lieu si elle était formée par un gaz étranger dont la dissolution serait évidemment favorisée par l'agitation.

L'expérience se fait facilement dans un tube en $U(f i g .2)$ con-

Fig. 2.

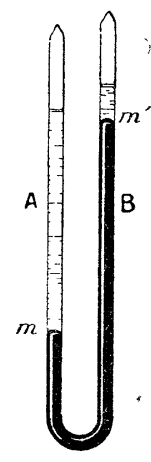

tenant du mercure et une certaine quantité de protoxyde d'azote liquéfié réparti dans les deux branches. Les pressions en $\mathrm{A}$ et en $\mathrm{B}$ étant égales à la tension maxima du protoxyde liquide, le mercure tend à se mettre au même niveau de part et d'autre et ce résultat peut en effet s'obtenir; mais, si les choses sont disposées comme l'indique la figure, on voit que, même s'il n'y avait plus de vapeur en $\mathbf{A}$, la dénivellation $\mathrm{mm}^{\prime}$ subsisterait encore. Sous l'influence de l'excès de pression qui en résulte pour la branche $\mathrm{A}$, la liquéfaction totale y a lieu invariablement. 
Inversement, si, dans la branche $\mathrm{B}$, dans laquelle le mercure est plus élevé, on condense par refroidissement toute la vapeur, le liquide, bien que soumis à une pression inférieure à sa tension maxima qui s'exerce alors en A, n'entre pas en ébullition, et le tube abandonné à lui-même reste indéfiniment dans le même état malgré les variations de la tempéralure ambiante.

En chauffant un peu le liquide dans la branche où il est condensé totalement, on le voit se dilater notablement sous pression constante sans bouillir.

Dans un tube relié à un manomètre, j'ai réussi à abaisser la pression de près de 10 atmosphères au-dessous de la tension maxima sans provoquer l'ébullition. Ce retard est surtout facile à obtenir avec l'acide carbonique, et, vers o', j'ai pu constater que la vaporisation, dans un tube ouvert avec précaution à l'air libre, se faisait uniquement par la surface; vers la fin de l'expérience seulement, le liquide résiduel s'est vaporisé brusquement en projetant dans tout le tube de la neige carbonique.

Ces expériences ne réussissent bien que dans des tubes parfaitement lavés, et s'ils doivent contenir du mercure ce dernier doit être bouilli dans le vide avant l'introduction du gaz liquéfié.

Avant d'aborder l'étude des phénomènes qui se produisent au voisinage du point critique, il était nécessaire de connaître ce point, au moins approximativement, pour le protoxyde d'azote pur. Une limite supérieure de la température cherchée m'a été fournie par les densités du liquide et de sa vapeur saturée ( $\left.{ }^{1}\right)$. Je rappellerai seulement que ces données ont été obtenues à $0^{\circ}$ par des pesées directes faites sur plusieurs grammes de matière; audessus de $0^{\circ}, j^{\prime}$ ai mesuré les volumes occupés par une masse connue de liquide entièrement séparé de sa vapeur, mais soumis à la tension maxima de celle-ci. Enfin, les densités de vapeur se déduisaient do l'observation du niveau dans un tube de Natterer rempli à peu près au volume critique, porté à diverses températures et agité plusieurs fois avant toute mesure. Le tube était divisé et jaugé; une pesée faisait connaître le poids de protoxyde d'azote contenu.

(') Comptes rendus des séances de l'Académie des Sciences, t. CXVIII, p. $1096 ; 189$ '. 
Une relation du premier degré donnait ensuite les densités de la vapeur en fonction de celles du liquide déjà déterminées.

Par ces dispositions j'ai pensé être à l'abri des phénomènes singuliers découverts par M. Gouy (1) et obtenir, aux erreurs de mesure près, la densité réelle du liquide et celle de la vapeur exactement saturée.

Les résultats obtenus m'ont conduit à admettre pour température critique approchée $+38^{\circ}, 8$. Cette valeur est sans doute trop élevée, mais, à la suite des travaux de M. Chappuis (loc. cit.), j'ai abandonné cette détermination qui me paraît pouvoir être plus rigoureusement faite par l'étude des indices qu'autrement.

Je me suis alors borné à établir qu'au-dessus de la température critique, par suite à $+38^{\circ}, 8$, le protoxyde d'azote se présentait comme un fluide parfaitement homogène. J'ai rempli à peu près au volume critique deux tubes en $\mathrm{O}$ contenant l'un du mercure, l'autre de l'acide sulfurique, et semblables à celui employé par MM. Cailletet et Colardeau (loc. cit.), mais scellés et indépendants. Le protoxyde liquide ayant été réuni d'abord dans l'une des branches où il formait une colonne de $25^{\mathrm{cm}}$, l'appareil a été porté à $+38^{\circ}, 8$. A cette température, les densités se sont montrées parfaitement égales, le mercure ou l'acide sulfurique ne présentant plus aucune différence de niveau. Par un refroidissement uniforme, la condensation s'est produite avec une simultanéité parfaite dans les deux branches, débutant par un nuage bleu dont la lumière était entièrement polarisée, et auquel succédait brusquement une véritable émulsion blanche formée de gouttelettes liquides; l'expérience terminée, des quantilés 'rigoureusement égales de liquide se présentaient dans les deux branches.

Un tube en U renversé, à courbure capillaire, analogue à celui dont se servait M. Battelli (loc. cit.), a donné la même identité de condensation après avoir été porté à $+38^{\circ}, 8$.

Il m'a paru indispensable de répéter les mêmes expériences avec l'acide carbonique dont le point critique vrai a été très exactement déterminé par M. Amagat au moyen des isothermes et par M. Chappuis en se servant de l'indice de réfraction; les deux

(1) Comptes rendus des séances de l'Académie des Sciences, t. CXVI, p. $1289 ; 1893$. 
nombres obtenus étant très voisins, il semblait d'autant plus intéressant de vérifier l'égalité des densités à la température pour laquelle l'égalis: ion des indices et la disparition de la partic rectiligne des isothermes ont lieu en même temps.

Avec le tube en O, aussi bien qu'avec l'appareil de M. Battelli, j'ai obtenu les mêmes résultats que précédemment, et à la température de $31^{\circ}, 7$, c'est-à-dire très peu au-dessus du point critique fixé à $3 \mathrm{I}^{\circ}, 4$ par M. Chappuis.

Il est facile de se rendre compte de la nécessité qu'il y a à employer dans ces expériences des gaz très purs.

Supposons, par exemple, que l'acide carbonique employé contienne à la pression ordinaire $\frac{1}{1000}$ d'air en volume ; ramené par la compression dans le tube en $\mathrm{O}$ à un volume environ 5oo fois plus faible, le volume critique, ce mélange contiendra de l'air à une pression voisine d'une demi-atmosphère, en le supposant également réparti dans l'appareil.

Par élévation de température, le liquide va entrer en ébullition, et l'air se réunira par distillation dans la branche qui ne contient que de la vapeur : sa pression y atteindra près d'une atmosphère. Au point critique, les densités en seront peu affectées, il est vrai, mais par refroidissement la condensation ne sera pas simultanée dans les deux branches, la vapeur n'étant pas saturée, dans celle qui renferme l'air, à la même température que dans l'autre et la différence des tensions étant voisine d'une atmosphère.

Or, on peut considérer que de l'acide carbonique ne renfermant que $\frac{1}{1000}$ d'air est beaucoup plus pur que celui qui a été employé dans la plupart des cas, même par Andrews. Ce titre de $\frac{999}{1000}$ n'a été dépassé que dans des expériences récentes. Quant au protoxyde d'azote, il renferme fréquemment 5 à ro pour ioo d'azote, dont il est impossible de le débarrasser par les moyens ordinaires.

Il n'est donc pas surprenant que les résultats que j'ai obtenus n'aient pas pu être constatés antérieurement.

On peut remarquer en passant que MM. Cailletet et Colardeau admettaient comme température critique la température de disparition du niveau dans un tube observé à l'œil nu. Ce phénomène paraît avoir lieu au-dessous du point critique vrai, comme l'a montré M. Pellat ( ${ }^{1}$ ), et la différence serait de quelques dixièmes

(1) Journal de Physique, 3e série, t. I, p. 225; 1892. 
de degré avec l'acide carbonique que j'ai préparé. On peut dès lors dire que l'état liquide persiste dans cet intervalle de température qui sera peut-être réduit par de nouvelles expériences.

J'ai tenu seulement à établir que l'égalisation réelle des densités avait bien lieu au delà du point critique vrai, ainsi que cela résulte de la théorie d'Andrews. Ce fait n'avait pas été vérifié directement avec les gaz.

Relativement à l'acide carbonique, j'ai cru devoir compléter ces expériences en étudiant les propriétés que peut mettre en évidence la coloration produite par l'iode et employée dans le même but par MM. Cailletet et Colardeau.

J'ai préparé un tube Natterer rempli d'acide carbonique renfermant une trace d'iode, et dont le volume était légèrement supérieur au volume critique afin de n'avoir que de la vapeur au point critique.

En agitant le tube pour favoriser la vaporisation ou la condensation, on observe qu'à la température ordinaire le liquide est fortement coloré, la vapeur très peu. Les deux colorations se rapprochent l'une de l'autre quand la température s'élève, et quand on dépasse $+3 \mathrm{I}^{\circ}$ elles paraissent absolument identiques, montrant ainsi que le liquide et sa vapeur, bien que séparés encore par un niveau, ont des propriétés à peine différentes et tendent vers un état unique.

Si le tube n'est pas agité, la coloration reste localisée à la partie inférieure, même à $+35^{\circ}$; elle tend seulement à se diffuser lentement. Par refroidissement, la condensation commence dans le bas du tube, contrairement à ce qui se passe dans l'expérience précédente ou dans un tube ne contenant pas d'iode, le phénomène se produisant alors partout à la fois.

Pour expliquer ce résultat, j'ai fait déposer sur les parois, par refroidissement à $-40^{\circ}$, l'iode peu soluble à cette température : en retournant le tube, le dépôt était en contact avec la vapeur seule, le liquide restant faiblement teinté. Dans ces conditions, j'ai élevé la température sans agiter. La vapeur carbonique dissolvant l'iode n'a pas tardé à être plus colorée que le liquide; en même temps, la vapeur qui se dégageait de ce dernier était absolument incolore et formait au-dessus du niveau une couche bien nette qui allait en se diffusanı dans le fluide de plus en plus co- 
loré qui la surmontait. Au-dessus de la température critique, cette zone incolore n'existait plus, ce qui semble indiquer qu'il n'y a plus alors de vaporisation. Par refroidissement, la condensation commençait dans le haut du tube, c'est-à-dire dans la partie la plus chargée d'iode. On comprend alors pourquoi, dans l'expérience précédente, la coloration restait localisée dans la partie occupée primitivement par le liquide : ce dernier en effet n'entraînait pas l'iode en se vaporisant. En même temps, la condensation n'étant pas homogène, on pouvait supposer qu'il existait encore du liquide à $+3 \check{5}^{\circ}$; la dernière expérience montre que c'est là une perturbation due à l'inégale répartition du corps dissous.

L'examen spectroscopique ne m'ayant montré, dans aucun cas, les cannelures caractéristiques du spectre de l'iode en vapeur, j'ai jugé nécessaire de faire une expérience à ce sujet avec un tube dans lequel il n'y avait plus de liquide à $+25^{\circ}$. J'étais ainsi en présence d'acide carbonique certainement gazeux à partir de cette température. J'ai donné au tube un diamètre intérieur de $8^{\mathrm{mm}}$ pour compenser le peu d'intensité de la coloration. En même temps, j'observais par comparaison un tube semblable renfermant de l'air à la pression ordinaire et un peu d'iode : en chauffant un peu ce dernier, j'obtenais une teinte en apparence identique à celle que présentait le tube à acide carbonique; elle suffisait largement pour voir le spectre sillonné de cannelures bien nettes, tandis que rien de semblable ne se manifestait avec l'acide carbonique coloré, aussi bien à $+40^{\circ} q u^{\prime a ̀}+2 \check{J}^{\prime \prime}$. Il faut donc admettre que l'iode dissous ainsi dans un gaz comprimé n'est pas simplement mélangé à l'état gazeux, et l'on comprend alors pourquoi le tube à acide carbonique présente, à égalité de température, une coloration beaucoup plus intense que celle du tube de comparaison. On voit également que l'absence des cannelures caractéristiques dans le spectre ne permet pas de conclure à la persistance de l'état liquide au delà du point critique.

Il n'y a par conséquent rien, dans ces expériences, qui soit en contradiction avec les idées d'Andrews, c'est-à-dire qui puisse infirmer l'existence, au-dessus du point critique, d'un fluide unique et parfaitement homogène.

J'ajouterai, pour terminer cette étude, que l'examen des courbes d'Andrews fournit une explication simple des résultats obtenus 
WYROUBOFF. - CORPS A DOUBLE POUVOIR ROTATOIRE. 451 par MM. Cailletet et Colardeau dans leurs expériences sur le point critique de l'eau ( ${ }^{1}$ ), expériences qu'ils avaient réalisées antérieurement avec l'acide carbonique (loc. cit.). A l'interprétation très simple donnée par M. Pellat $\left({ }^{2}\right)$ je crois pouvoir ajouter une simple remarque.

Si, dans le diagramme donné au début de cet exposé, on considère plusieurs lignes d'échauffement à volume constant, telles que $V_{1} V_{1}^{\prime}, V_{2} V_{2}^{\prime}$, ces lignes, sans avoir besoin d'être très voisines de l'ordonnée $V_{c} V_{c}^{\prime}$ qui correspond au volume critique, rencontreront l'isotherme critique sensiblement à la même hauteur, par suite à la même pression $\mathrm{P}_{c}$, et cela en raison de l'inflexion assez allongée de l'isotherme. Au point critique, la pression sera donc sensiblement la même pour plusieurs volumes différents, ou encore pour plusieurs masses occupant le même volume. Il n'en sera évidemment plus de même aux températures plus élevées, comme le montre la $f i g$. I.

Les courbes de tension maxima resteront donc confondues jusqu'à l'isotherme critique, au moins pratiquement, et ne se sépareront qu'au delà, à une température d'autant plus élevée que le manomètre employé sera moins sensible. Elles formeront alors le faisceau divergent partant assez exactement d'un point unique, et si nettement obtenu par les expérimentateurs qui ont imaginé cetle ingénieuse méthode. 